\title{
Editorial: Carbohydrates: the yet to be tasted sweet spot of immunity
}

\author{
Deirdre R. Coombe ${ }^{1 *}$ and Christopher R. Parish ${ }^{2}$ \\ ${ }^{1}$ School of Biomedical Sciences, CHIRI Biosciences Research Precinct, Faculty of Health Sciences, Curtin University, Perth, \\ WA, Australia, ${ }^{2}$ Cancer and Vascular Biology Group, Department of Cancer Biology and Therapeutics, John Curtin School of \\ Medical Research, The Australian National University, Canberra, ACT, Australia
}

Keywords: Siglec, sialic acid, glycan, galectin, glycosaminoglycan, hyaluronan, heparan sulfate, heparanase

\section{OPEN ACCESS}

Edited by:

Pietro Ghezzi,

Brighton and Sussex Medical School,

UK

Reviewed by:

Alex Langford-Smith,

The University of Manchester, UK

*Correspondence:

Deirdre R. Coombe

d.coombe@curtin.edu.au

Specialty section:

This article was submitted to Inflammation, a section of the journal

Frontiers in Immunology

Received: 27 May 2015 Accepted: 02 June 2015 Published: 17 June 2015

Citation: Coombe DR and Parish CR (2015) Editorial: Carbohydrates: the yet to be tasted sweet spot of immunity. Front. Immunol. 6:314.

doi: 10.3389/fimmu.2015.00314
Glycobiology is an expanding discipline. Nowhere is this more apparent than in our understanding of the immune response. Perhaps the title of this focused research topic should be: "Carbohydrates: now the sweet spot of immunity!" The revolution in thinking to embrace the "glyco" component of glycoproteins and glycolipids has been accompanied by the development of new technologies that allow the structure of many different glycans to be determined. The article by van Kooyk et al. provides an introduction to glycan analytical tools (1). These range from technically simple analyses using plant lectins combined with flow cytometry or ELISA methods to obtain clues of glycan structures, to more complex sequencing methodologies for detailed structural characterizations. Nevertheless, determining the structure of some glycans, and particularly the glycosaminoglycans (GAGs), is still extremely difficult. However, good progress is being made in this area (2).

Cell surface glycosylation is a characteristic of all living cells $(3,4)$, thus it is logical that glycan structures are involved in self or non-self recognition. Nevertheless, glycans have been excluded from the thinking of most immunologists. Probably a lack of appreciation of the specificity of carbohydrate-protein interactions and the diversity of glycan structures led to this outcome. Yet, it is glycan diversity that has been harnessed by microbes to coat their surfaces, and most immunogens on microbes are glycans. As pathogens developed their glycan coats their vertebrate and invertebrate hosts similarly developed molecules to recognize these structures. The idea that invertebrate lectins can recognize glycan structures on microbes, thereby facilitating microbe phagocytosis, was accepted decades ago (5), but the fundamental contribution of glycan-protein interactions to mammalian immunity was accepted only recently. Numerous molecules involved in invertebrate host defense that recognize a spectrum of glycan structures on bacteria, fungi, and other pathogens are clearly related to similarly acting proteins in modern mammals (6). The lectin pathway of complement, toll-like receptors, the pentraxin pattern recognition receptors, and the galectins all probably arose initially in invertebrates ancestors and had roles in self or non-self recognition. We now know glycans and their binding proteins contribute to all aspects of immunology. It was argued that the essential role glycan-protein binding events play in host defense and infection is the driver of glycan diversity $(3,4)$. The evolutionary selection pressures imposed by the need of pathogens to avoid recognition by the proteins of their host's immune system, and for hosts to rapidly evolve glycan structures that are not sites for pathogen adhesion and infection, it was proposed, led to the conservation of glycan structural diversity $(3,4)$.

An appreciation of carbohydrate structural diversity is obtained when the number of genes involved in glycan biosynthesis is appreciated. van Kooyk et al. revealed that if all the genes involved in glycan biosynthesis are considered they would comprise around 3-4\% of the genome (1). Although they primarily encode enzymes, co-factors, transporters, and activated sugar donors are also involved. Regulation of the expression of these genes, regulation of the activity of the different glycosyltransferases through a diverse collection of mechanisms, coupled with regulation of the expression of core proteins adds an extra dimension to glycoconjugate structural diversity (1). 
Given glycan biosynthetic processes, it is not surprising that glycan structures are altered in response to physiological and pathological cues, and these different structures affect the immunological outcomes of the process in which they are involved.

Dendritic cell (DC) sialic acids illustrate how glycan structures can influence the adaptive immune response. Glycans, both $\mathrm{N}$ - and $\mathrm{O}$-linked, on glycoproteins terminate in sialic acid, and glycolipid gangliosides contain one or more sialic acids. Sialic acids shield host cells from pathogens, prevent the deposition of complement components on DCs, and interact with receptors of the Siglec and Selectin families (7). As explained by Crespo et al., the concentration of sialic acids on DCs is very high, most Siglecs binding to sialic acids on the same DC (i.e., cis interactions) (7). Sialidase activity releases these Siglecs allowing them to engage in interactions with sialic acids on pathogens. The balance between cell surface sialic acid and sialidases may regulate key DC functions like phagocytosis, micropinocytosis, migration, and DC-T cell interactions (7). Not all leukocytes can have these high-sialic acid levels, nor can their Siglecs signal via sialic acid in cis interactions. As the binding of Siglecs on eosinophils and neutrophils to antibodies or multimeric glycan ligands triggers cell death (8), yet in certain inflammatory conditions, these cell types abound; this could not happen if these cells have high-sialic acid levels that bind Siglecs in cis to trigger cell death. Nevertheless, inhibitory intracellular signals upon Siglec binding sialylated antigens are common, because most Siglecs have inhibitory ITIM signaling motifs and DCs may become tolerogenic if their Siglecs recognize sialylated carbohydrate antigens in tumors (7).

Dendritic cell immunogenicity is also regulated by other carbohydrate-protein interactions; the interaction of galectin-1 with DCs encourages a tolerogenic phenotype (9). Galectins are a family of $\beta$-galactoside binding lectins. Various galectin family members have been described as "regulators of immune homeostasis," as "pattern recognition receptors", and as "receptors for microbial adhesion and infection" (10). Often there is evidence for the same galectin having opposing functions, the question is how? Baum et al. examined the opposing roles of galectins in microbe-host interactions (11). They described how galectins can bridge specific glycans on viral and bacterial pathogens with glycans on target cell plasma membranes, to increase pathogen attachment. The outcome of galectin-pathogen interactions is not always infection; rather there are numerous examples of galectins contributing to innate and adaptive immune responses to pathogens, and some galectins have direct microbicidal activity (11). The response is dependent on the galectin, the pathogen and the host cell, with factors such as glycan density, glycan clustering, and the glycoprotein or glycolipid upon which the glycan is presented, all contributing to the context-specific outcome. Differences in the $N$-glycans of resting and activated cytolytic T lymphocytes (CTLs), with more galectin-3 ligands being present on activated CTLs, is an example where the density of a glycan structure regulates CTL function. In a galectin-3 rich milieu (e.g., a tumor), reduced motility of galectin- 3 cross-linked glycoproteins on activated infiltrating CTLs could explain the decreased CTL activity within tumors (12).

Involvement in the immune response is also in the functional realm of GAGs. Simon Davis and Parish highlight the number of proteins that have heparin/heparan sulfate (HS) binding motifs within their sequences (13). Many of the possible new HS-protein interactions that they discovered may act in immune responses but this is unconfirmed. Other confirmed HS-protein interactions have clear implications for immunity; described are examples of HS-protein interactions contributing to (1) cell adhesion and migration, (2) the regulation of cytokine and chemokine functions, and (3) the sensing of tissue injury (13). The regulation, by HS, of complement pathway triggered inflammation is emphasized by two articles. Perkins et al. used molecular modeling and affinity coefficient data to develop a bivalent, co-operative model of complement factor $\mathrm{H}(\mathrm{CFH})$ binding to HS (14). They argued, mutations in either of the $\mathrm{CFH}$ HS binding regions that weaken binding, alters the orientation of $\mathrm{CFH}$ on the cell surface disrupting $\mathrm{C} 3 \mathrm{~b}$ binding and the regulation of $\mathrm{C} 3 \mathrm{~b}$ activity, with the result being inflammatory damage, whereas, Clark et al. offered the opinion that different HS structures (or "postcodes") in the glycomatrix of different tissues determine the levels of immobilized CFH. Probably, both explanations apply and collectively they explain the disease association of $\mathrm{CFH}$ polymorphisms (15).

The association of GAGs with inflammation extends beyond complement pathway regulation. Chemokine-HS interactions are known to establish chemokine gradients to direct leukocytes to inflammatory sites (16); but the contribution of the HS enzyme, heparanase (Hpse), to inflammatory disease is under appreciated. Heparanase assists leukocyte migration across basement membranes by acting as a "path-maker"; however, in type 1 diabetes Hpse activity actually drives the disease process (17). Simeonovic et al. describe how within pancreatic islets there are extraordinarily high levels of HS; this HS is essential for beta-cell survival. If active Hpse degrades HS in the islet basement membrane, inflammatory mononuclear cells can enter the islet; Hpse from these cells destroys intra-islet HS, triggering beta-cell death, and destructive insulitis. The ubiquitous non-sulfated GAG, hyaluronan (HA) is also involved in inflammation. Normally, it has a very high-average molecular weight, but at sites of inflammation and tissue injury HA polymers of overlapping length and function occur. As explained by Petrey and de la Motte, HA can promote and suppress inflammation, functions that depend upon polymer length and the activities of HA-binding proteins (18). The ability of hyaluronidases to degrade HA depends on the conformation of HA chains, which is influenced by the degree and hierarchy of protein-HA interactions, both of which depend on the HA-binding proteins in the microenvironment (18). The tissue microenvironment, its carbohydrates and their binding proteins, underpins the regulation of inflammation by $\mathrm{HA}$ and $\mathrm{HS}$ in a range of diseases including type 1 diabetes $(18,19)$.

The contribution of HS to human immunodeficiency virus (HIV-1) infection has come of age. Connell and Lortat-Jacob indicate how the elegant design of a potential drug developed through an appreciation of the molecular events involved in HIV-1 infection of CD4+ leukocytes (20). Although the surface exposed V3 loop of the virus protein, gp120, is involve in HS binding, prior CD4 binding was found to induce a HS binding site that is also involved in binding to HIV-1's co-receptors, CXCR4 or CCR5. The glycoconjugate drug candidate was designed to block HIV from 
binding to cell surface HS, its co-receptor and CD4. It is composed of a small CD4 mimetic linked to a chemically synthesized HS dodecamer (20). This glycoconjugate had strong anti-viral activity against HIV-1 regardless of its co-receptor usage, which is a major advance.

These articles highlight the contribution of glycans to different aspects of the immune response, yet this is a "taster plate" of their total contribution. Contrary to the often held view, glycan structures frequently bind proteins with quite exquisite specificity; our lack of understanding of their binding modes and the nature of the protein conformations that are recognized cause the miss-interpretation. Reductionist thinking and analyses, although

\section{References}

1. van Kooyk Y, Kalay H, Garcia-Vallejo JJ. Analytical tools for the study of cellular glycosylation in the immune system. Front Immunol (2013) 4:451. doi:10.3389/ fimmu.2013.00451

2. Kailemia MJ, Ruhaak LR, Lebrilla CB, Amster IJ. Oligosaccharide analysis by mass spectrometry: a review of recent developments. Anal Chem (2014) 86:196-212. doi:10.1021/ac403969n

3. Gagneux P, Varki A. Evolutionary considerations in relating oligosaccharide diversity to biological function. Glycobiology (1999) 9:747-55. doi:10.1093/ glycob/9.8.747

4. Varki A. Evolutionary forces shaping the Golgi glycosylation machinery: why cell surface glycans are universal to living cells. Cold Spring Harb Perspect Biol (2011) 3:a005462. doi:10.1101/cshperspect.a005462

5. Coombe DR, Ey PL, Jenkin CR. Self-non-self recognition in invertebrates. Q Rev Biol (1984) 59:231-56. doi:10.1086/413901

6. Iwanaga S, Lee BL. Recent advances in the innate immunity of invertebrate animals. J Biochem Mol Biol (2005) 38:128-50. doi:10.5483/BMBRep.2005.38. 2.128

7. Crespo HJ, Lau JT, Videira PA. Dendritic cells: a spot on sialic acid. Front Immunol (2013) 4:491. doi:10.3389/fimmu.2013.00491

8. Bochner BS, Zimmermann N. Role of Siglecs and related glycan-binding proteins in immune responses and immunoregulation. J Allergy Clin Immunol (2015) 135:598-608. doi:10.1016/j.jaci.2014.11.031

9. Mascanfroni ID, Cerliani JP, Dergan-Dylon S, Croci DO, Ilarregui JM, Rabinovich GA. Endogenous lectins shape the function of dendritic cells and tailor adaptive immunity: mechanisms and biomedical applications. Int Immunopharmacol (2011) 11:833-41. doi:10.1016/j.intimp.2011.01.021

10. Vasta GR, Ahmed H, Nita-Lazar M, Banerjee A, Pasek M, Shridhar S, et al. Galectins as self/non-self recognition receptors in innate and adaptive immunity: an unresolved paradox. Front Immunol (2012) 3:199. doi:10.3389/fimmu. 2012.00199

11. Baum LG, Garner OB, Schaefer K, Lee B. Microbe-host interactions are positively and negatively regulated by galectin-glycan interactions. Front Immunol (2014) 5:284. doi:10.3389/fimmu.2014.00284

12. Antonopoulos A, Demotte N, Stroobant V, Haslam SM, van der Bruggen P, Dell A. Loss of effector function of human cytolytic T lymphocytes is useful, in isolation are unlikely to reveal the truth. Repeatedly, it is the "context," whether the presentation of glycan motifs, or the molecules (proteins and carbohydrates) of the surrounding microenvironment, which determines the outcome of glycan-protein interactions. It is fitting that the concluding article in this series (20) describes the development of a glycan inspired potential therapeutic, because this is an area of drug discovery currently under exploited. Advances in technologies of glycan structure determination and syntheses, coupled with a more holistic approach to understanding glycan interactions with their binding partners will lead to more glycan inspired therapeutics to treat immunological diseases.

accompanied by major alterations in $\mathrm{N}$ - and O-glycosylation. J Biol Chem (2012) 287:11240-51. doi:10.1074/jbc.M111.320820

13. Simon Davis DA, Parish CR. Heparan sulfate: a ubiquitous glycosaminoglycan with multiple roles in immunity. Front Immunol (2013) 4:470. doi:10.3389/ fimmu.2013.00470

14. Perkins SJ, Fung KW, Khan S. Molecular interactions between complement factor $\mathrm{H}$ and its heparin and heparan sulfate ligands. Front Immunol (2014) 5:126. doi:10.3389/fimmu.2014.00126

15. Clark SJ, Bishop PN, Day AJ. The proteoglycan glycomatrix: a sugar microenvironment essential for complement regulation. Front Immunol (2013) 4:412. doi:10.3389/fimmu.2013.00412

16. Tanino Y, Coombe DR, Gill SE, Kett WC, Kajikawa O, Proudfoot AE, et al. Kinetics of chemokine-glycosaminoglycan interactions control neutrophil migration into the airspaces of the lungs. J Immunol (2010) 184:2677-85. doi:10. 4049/jimmunol.0903274

17. Simeonovic CJ, Ziolkowski AF, Wu Z, Choong FJ, Freeman C, Parish CR Heparanase and autoimmune diabetes. Front Immunol (2013) 4:471. doi:10. 3389/fimmu.2013.00471

18. Petrey AC, de la Motte CA. Hyaluronan, a crucial regulator of inflammation. Front Immunol (2014) 5:101. doi:10.3389/fimmu.2014.00101

19. Bogdani M, Korpos E, Simeonovic CJ, Parish CR, Sorokin L, Wight TN. Extracellular matrix components in the pathogenesis of type 1 diabetes. Curr Diab Rep (2014) 14:552. doi:10.1007/s11892-014-0552-7

20. Connell BJ, Lortat-Jacob H. Human immunodeficiency virus and heparan sulfate: from attachment to entry inhibition. Front Immunol (2013) 4:385. doi:10.3389/fimmu.2013.00385

Conflict of Interest Statement: The authors declare that the research was conducted in the absence of any commercial or financial relationships that could be construed as a potential conflict of interest.

Copyright $(2) 2015$ Coombe and Parish. This is an open-access article distributed under the terms of the Creative Commons Attribution License (CC BY). The use, distribution or reproduction in other forums is permitted, provided the original author(s) or licensor are credited and that the original publication in this journal is cited, in accordance with accepted academic practice. No use, distribution or reproduction is permitted which does not comply with these terms. 